\title{
From random to self-organized clusters on surfaces: a variable temperature STM study
}

\author{
S. Padovani, I. Chado, F. Scheurer, and J.-P. Bucher
}

IPCMS, Groupe Surfaces Interfaces, UMR 7504, CNRS-ULP, 23 rue du Loess, 67037 Strasbourg Cedex, France

The spontaneous organization of clusters of adsorbed metal atoms on surfaces is driven by the substrate temperature imposed during growth. The morphology and distribution of nanostructures can be controlled: from very small (a few atoms) randomly distributed clusters to bigger (several 100 atoms) self-organized clusters on a regular network. From a detailed variable temperature Scanning Tunneling Microscopy (STM) study, it is suggested that this way of organizing matter at the nanoscale may be used to achieve new material properties. In particular, on the example of Co on Au(111), it is shown that the direction of the magnetization can be adjusted from in-plane to out-of-plane, depending on the growth condition.

hysical and chemical properties of nanostructures on surfaces are influenced to a large extent by their morphologies and their surface density. Concomitantly, recent progress in spin electronics, magnetic data storage and sensors have proven to rely strongly on material properties at the nanoscale [1]. Fascinating phenomena are expected when characteristic dimensions of the nanostructures lead to confinement of electrons at a length scales where quantum coherence occurs. In order to get a deeper insight into the relationship between nanoscale structure and physical properties (in our case magnetism), we developed a new approach based on self-organization of magnetic clusters. In this approach surface reconstruction are used as templates to grow clusters with sizes between 10 and several 100 atoms. By means of variable temperature UHV scanning

${ }^{1}$ In a Scanning Tunneling Microscope (STM) the topographic image of a surface is obtained while scanning the surface with a sharp metal tip at a distance of a few Angstroms. The tunneling current that establishes between the tip and the sample is measured and kept constant by a feedback loop so that the tip follows the surface roughness at an atomic scale without being in contact with the surface. A piezo-crystal allows to perform the atomic scale movements in $x, y$ and $z$ directions. The voltage applied to the $z$ electrode of the piezo-crystal by the feedback loop, as a function of ( $x$, $y$ ), directly provides the topographic image of the surface. The big advantage of STM over other techniques is its ability to visualize individual atoms in direct space with a lateral resolution of less than an Angstrom and a high-resolution (in the $z$-direction) of less than a tenth of an Angstrom. It furthermore allows to perform spectroscopic measurements. For more details about STM see for example [2]. tunneling microscopy (STM) ${ }^{1}$, and in situ magneto-optic Kerr measurements (see Fig. 1), we establish a correspondence between morphology and magnetic properties of as grown nanostructures over a wide temperature range (down to $30 \mathrm{~K}$ ).

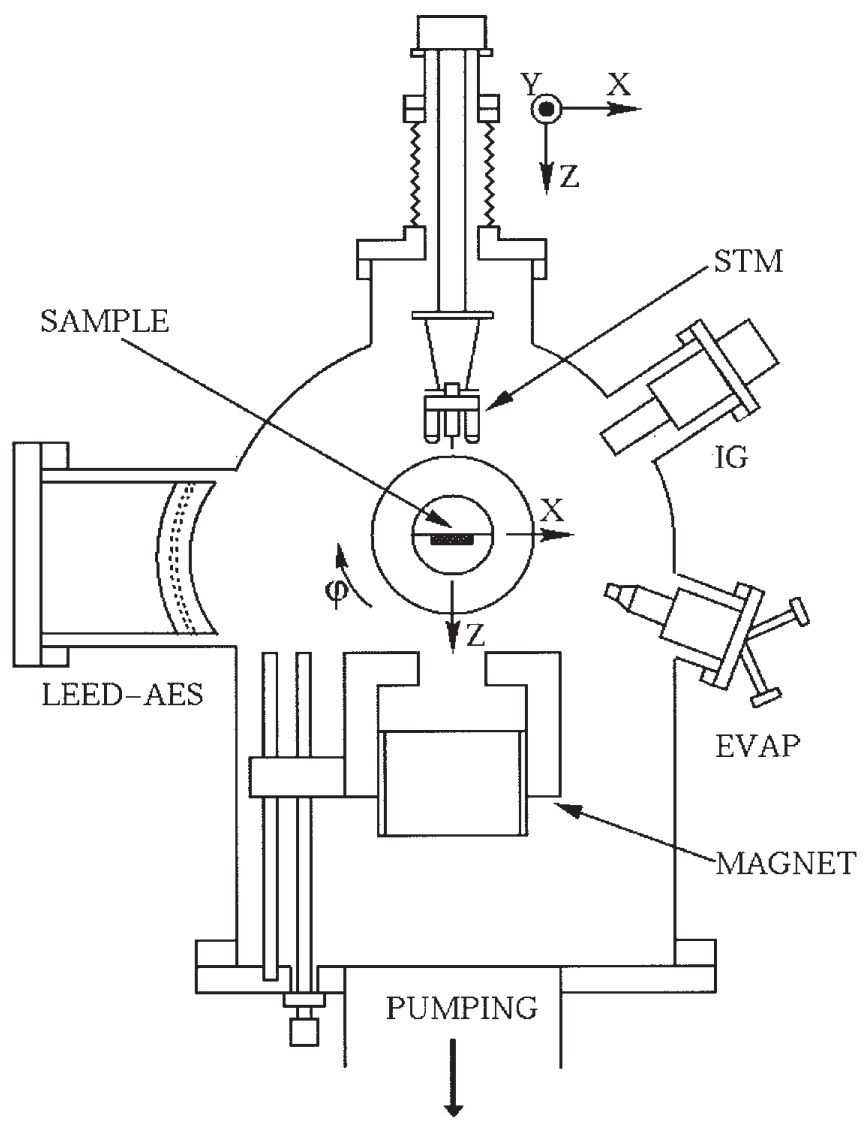

Figure 1. Variable temperature STM: The apparatus in use at IPCMS is a home built system consisting of one single ultrahigh vacuum (UHV) chamber. The sample is connected to a helium flux-cryostat, which allows to vary its temperature from $20 \mathrm{~K}$ up to several $100 \mathrm{~K}$. A beetle STM can be lowered down onto the sample holder. The apparatus is equipped with conventional UHV facilities such as ion gun, electron beam evaporator as well as LEED and Auger for crystallographic and chemical characterization. Magnetic properties of the deposit can be measured in situ by a magneto-optic Kerr (MOKE) probe by means of a removable magnet. The optics of the MOKE experiment is not shown in the picture. 


\section{Exploiting reconstruction and strain relaxation}

Self-organization of aggregates and islands on surfaces strongly relies on the occurrence of surface reconstruction. Some surfaces reconstruct spontaneously as a result of free energy minimization. This means that with respect to their positions in the bulk crystal, surface atoms adopt new equilibrium positions. An example of such a reconstruction is the chevron reconstruction of the $\mathrm{Au}(111)$ surface, that produces rearrangements of atoms on a mesoscopic scale (see Fig. 2).

Visible in figure $2 \mathrm{a}$ are the bright contrasts of the zigzag domain walls that produce corrugations in the STM images of about $0.2 \AA$. These so called discommensuration walls are limiting fcc and hep stacking domains of the topmost atomic layer which form spontaneously by strain relaxation (see Fig. 2b). The registry of surface gold atoms varies from hollow sites of the fcc to the hollow sites of the hep stacking (the fcc regions are wider than the hcp regions). The fcc to hcp transition (the soliton wall) appears as ridges in the STM images since surface atoms near bridge sites rest about $0.2 \AA$ higher than in hollow sites (Fig. 2a). Similar dislocation networks have been obtained on heteroepitaxial systems (see the case of Ag bilayers on $\mathrm{Pt}(111)$ [3]). The average period of the dislocation pattern is then given by $\mathrm{D}=\mathrm{a}_{\mathrm{film}} /\left(\mathrm{a}_{\mathrm{film}}-\mathrm{a}_{\text {substr }}\right)$, as a function of the lattice constants of film and substrate.

Due to its particular conformation, the zigzag reconstruction of $\mathrm{Au}(111)$ leads to singularities that can best be illus- trated as single point dislocations (for example five nearest neighbors instead of six) located at the elbows of the chevron reconstruction [4]. The dislocations form a rectangular lattice with a unit cell $75 \AA \times 140 \AA$. Although this reconstruction has first been evidenced experimentally, it is fairly well understood today thanks for example to molecular dynamics simulations [5].

\section{Nucleation on defects}

It has been observed that such surfaces may be used as templates to grow organized metal clusters $[4,6]$. The point dislocations at the elbows of the chevron reconstruction act as nucleation sites for atoms of cobalt adsorbed from the gas phase. At its initial stage, this mechanism involves a site exchange between one $\mathrm{Co}$ and one $\mathrm{Au}$ atom [7]. This substituted atom will then act as a nucleation site for further incoming Co atoms. In this way, self-organized cobalt bilayer clusters containing 200 atoms each, are synthesized (Fig. 3). If one would be able to store information in these dots, one would reach a storage density of $10^{12}$ bits $\times \mathrm{cm}^{-2}$ namely $10^{3}$ times higher than the highest storage densities reached today.

These clusters are stable in UHV environment up to $400 \mathrm{~K}$. Above this temperature, the clusters burrow into the gold substrate, by simultaneously expelling gold atoms. This is due to the low surface energy of gold which tends to encapsulate the cobalt clusters [8]. Provided the annealing is
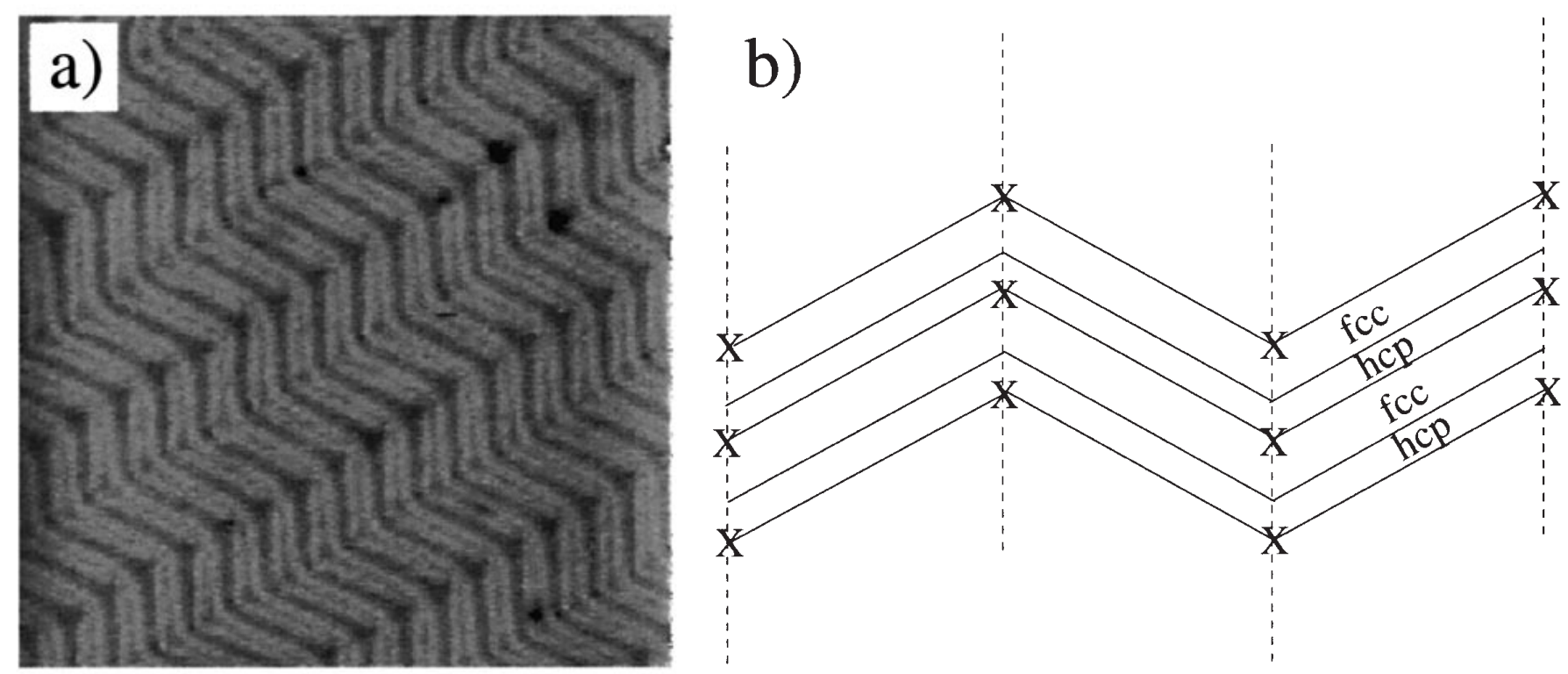

Figure 2. a) STM image of the zigzag reconstruction of the $A u(111)$ surface (image: $100 \mathrm{~nm} \times 100 \mathrm{~nm}$ ). b) Schematic view of the domains. Crosses indicate the location of point dislocations. 


\section{Dossier}

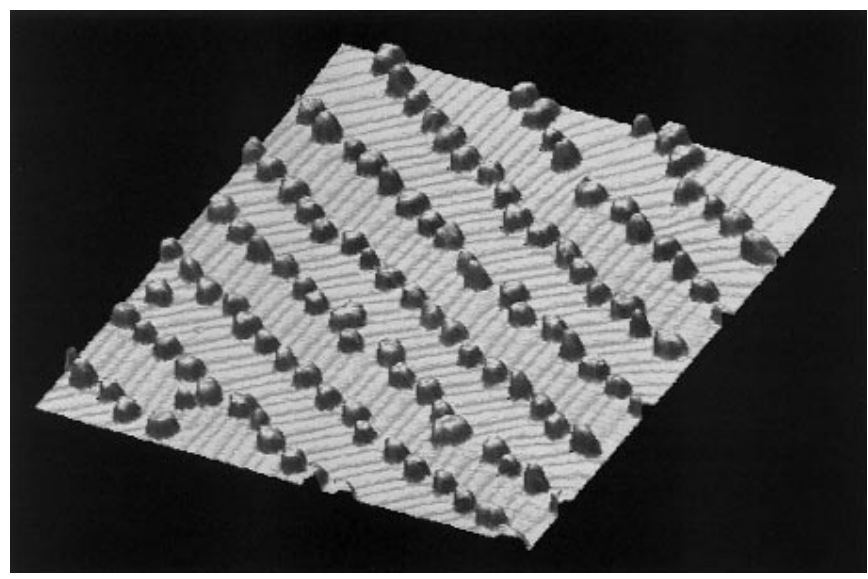

Figure 3. STM image $(100 \mathrm{~nm} \times 100 \mathrm{~nm})$ of self-assembled cobalt clusters, approximately 200 atoms each, on the zigzag reconstruction of the $A u(111)$. The separation between two clusters along a row is $75 \AA$, adjacent rows are separated by $140 \AA$.

performed below $600 \mathrm{~K}$, this procedure does not perturb significantly the cobalt clusters since no modifications of the magnetic properties are observed. For ex situ measurements and potential applications, Co clusters and films can be stabilized by a protective film of a few gold monolayers [9].

\section{Nucleation dictated by kinetics}

While at room temperature, the growth of Co is governed by the reconstruction of the $\mathrm{Au}(111)$ surface, at low temperature on the contrary, nucleation is dictated by kinetics and leads to completely different growth modes. The low temperature nucleation, was explored by variable temperature STM. At some point when lowering the substrate temperature, the mean free path of diffusion becomes smaller than the separation between point dislocations, the density of islands is then given by $N \sim R^{1 / 3} \exp \left(-E_{d} / 3 k T\right)$, with $R$ the deposition rate and $E_{d}$ the diffusion barrier for a cobalt adatom on the gold surface. As a result, a high density of islands in the early growth stage at low temperature is achieved. A dramatically different growth mode (Fig. 4a) then guarantees that the small monolayer thick islands coalesce well before the natural tendency of 3D growth sets in. A quasi layer-by-layer growth occurs contrary to the 3D island growth observed at $300 \mathrm{~K}$. As can be foreseen, the properties of films grown at low and high temperatures will be quite different and are illustrated now on the example of magnetism.

\section{Application: tuning the magnetic properties}

The magnetic properties of a deposit can be characterized by the magnetic anisotropy. It quantifies the tendency of the
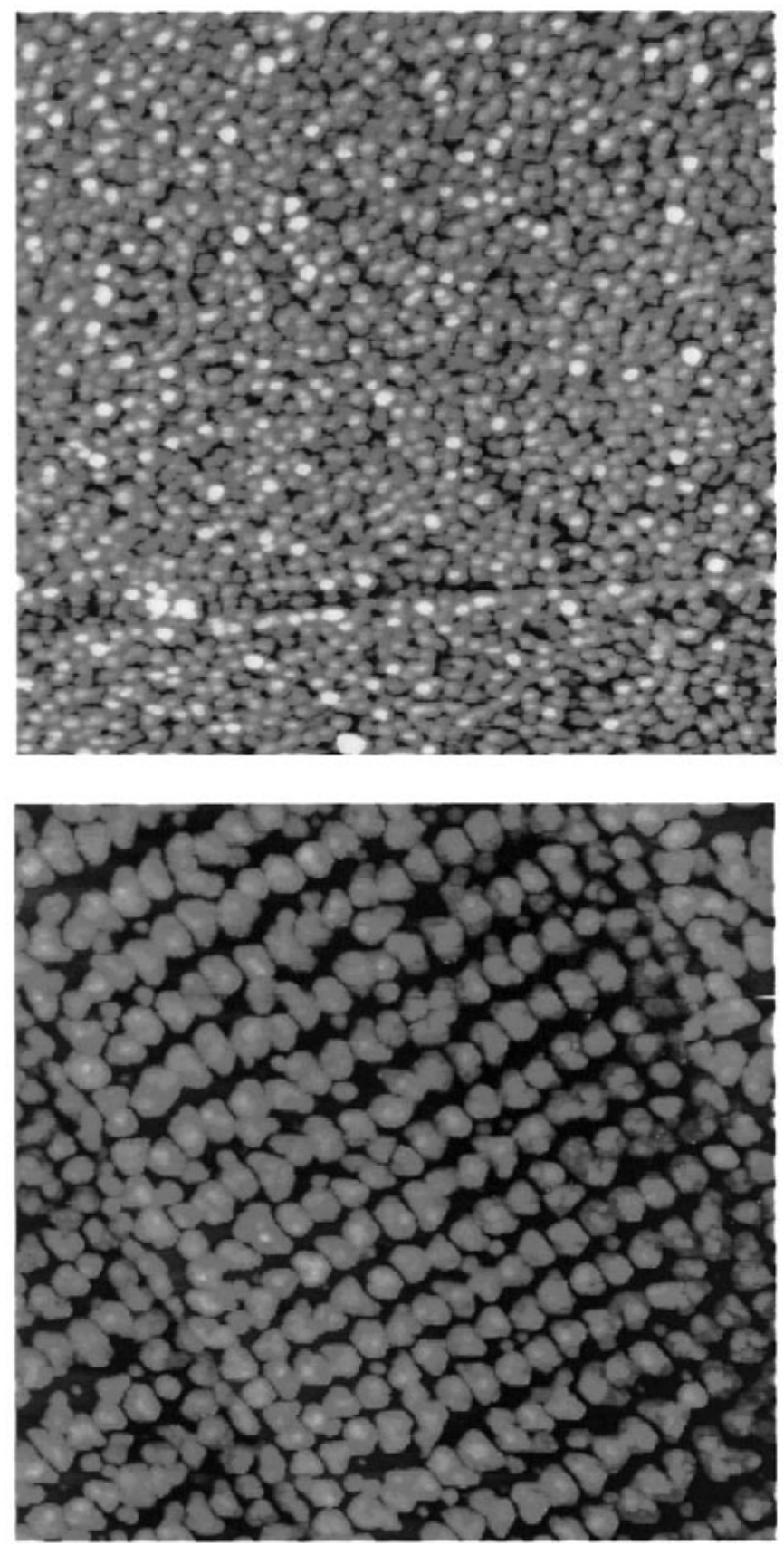

Figure 4. Comparison between films grown at: a) $30 \mathrm{~K}, 1.7 \mathrm{ML}$ $(100 \mathrm{~nm} \times 100 \mathrm{~nm})$ and b) $300 \mathrm{~K}, 1.0 \mathrm{ML}(150 \mathrm{~nm} \times 150 \mathrm{~nm})$. In the first case a compact stacking of monolayer thick clusters with a diameter of $0.5 \mathrm{~nm}$ occurs, while the growth at $300 \mathrm{~K}$ leads to bilayer clusters with a lateral dimension of $7 \mathrm{~nm}$.

magnetization to be aligned along a preferential axis. The magnetic anisotropy can be measured by recording hysteresis loops in a configurations where the probing field is applied successively in-plane and out-of-plane. Magnetization versus field measurements are done by 

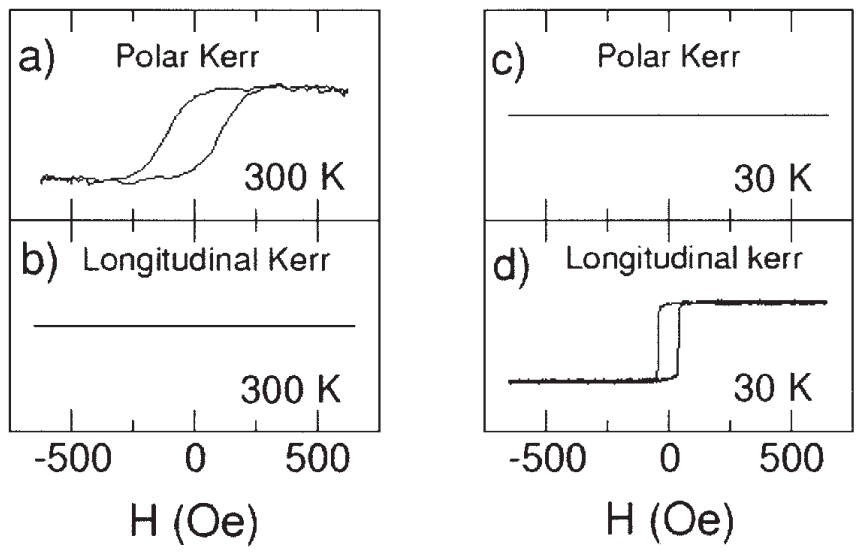

Figure 5. Polar and longitudinal hysteresis loops for 1.7-ML Co films on $\mathrm{Au}(111)$ deposited at $300 \mathrm{~K}$, (a) and (b); deposited at $30 \mathrm{~K}$, (c) and (d). After growth at $300 \mathrm{~K}$, a rectangular loop (a) is seen in the polar configuration but no signal in the longitudinal one (b) which is relevant for a perpendicular magnetization. The opposite is true for the films grown at $30 \mathrm{~K}$ and the magnetization lays in plane.

magneto-optic Kerr effect (MOKE). MOKE measures the rotation of the polarization of light due to the interaction with the magnetic structure, it has a probing depth of about $50 \mathrm{~nm}$; the results are summarized in figure 5. The structures obtained at $300 \mathrm{~K}$ are known to possess perpendicular (outof-plane) magnetic anisotropy for equivalent thicknesses of cobalt between 2 and 5 ML [10]. The perpendicular orientation of the magnetization is particularly attractive for magneto-optic data storage. For the films grown at $30 \mathrm{~K}$, on the contrary, we show that in-plane magnetization is stabilized. It is not the purpose of this paper to go into the details of the relation between topography and magnetism but the reader interested in magnetism can find all the necessary details in ref. [11]. As a conclusion, spin engineering becomes possible since continuous variation of the anisotropy from in-plane to out-of-plane is now feasible. Furthermore, as is shown in ref. [9], self-organized clusters form a model system to test magnetism at various dimensionalities: 0D, 1D and 2D.

\section{Acknowledgments}

This work has been supported by the Centre National de la Recherche Scientifique (CNRS-Ultimatech program).

\section{References}

1. Physics Today, April 1995 special issue on "Magnetoelectronics".

2. Scanning Tunneling Microscopy, Guntherodt, H.-J.; Wiesendanger, R. Eds, Springer: Berlin, 1992, Vol. I.

3. Brune, H.; Roeder, H.; Boragno Kern, C. K. Phys. Rev. 1994, B 49, 2997.

4. Chambliss, D.D.; Wilson, R.J.; Chiang, S. J. Vac. Sci. Technol. 1991, B9, 993.

5. Narasimhan, S.; Vanderbilt, D. Phys. Rev. Lett. 1992, 69, 1564.

6. Voigtländer, B.; Meyer, G. N.M. Amer, Phys. Rev. 1991, B44, 10354.

7. Meyer, J. A.; Baike, I. D.; Kopatzki, E.; Behm, R. J. Surf. Sci. 1996, 365, L647.

8. Padovani, S.; Scheurer, F.; Bucher, J.P. Europhys. Lett. 1999, 45, 327.

9. Padovani, S.; Chado, I.; Scheurer F.; Bucher, J.P. Phys. Rev. 1999, B59, 11887.

10. Chappert, C.; Bruno, P. J. Appl. Phys. 1991, 64, 5736.

11. Padovani, S.; Scheurer, F.; Chado, I.; Bucher, J.P. Phys. Rev. 2000, B61, 72 . 